\title{
Postoperative Komplikationen beim laparoskopischen Roux-Y-Magenbypass in der Adipositaschirurgie
}

\author{
Günther Meyer Christine Stier Oliver Markovsky \\ Abteilung für Allgemein-, Viszeral- und Gefäßchirurgie, Chirurgische Klinik München-Bogenhausen, München, Deutschland
}

\author{
Schlüsselwörter \\ Laparoskopischer Magenbypass - Adipositaschirurgie · Kom- \\ plikationen · Ergebnisse
}

\section{Zusammenfassung}

Der laparoskopische Roux-Y-Magenbypass weist eine Reihe charakteristischer chirurgischer Komplikationsmöglichkeiten neben den in der bariatrischen Chirurgie generell vorhandenen Risiken auf. Durch die minimal-invasive Technik konnte das Risiko insgesamt vermindert werden. Die Mortalität beträgt $0-0,5 \%$, das Morbiditätsrisiko beträgt zwischen 6 und $30 \%$. Dabei erschweren die speziellen Umstände des morbid adipösen Patienten häufig die Diagnosestellung, und insbesondere septische Komplikationen mit einem hohen Mortalitätsrisiko stellen eine große Gefahr dar. Größtmögliche Sicherheit und Prävention sind daher besonders wichtig. Die häufigsten Todesursachen sind Lungenembolie und Anastomoseninsuffizienz. Die häufigsten Komplikationen haben pulmonale und kardiale Ursachen. Wundkomplikationen werden als Folge des laparoskopischen Zugangs sehr selten beobachtet. Chirurgische Komplikationen resultieren im Wesentlichen aus Blutungen, Problemen an den Anastomosen und Nähten sowie dem Dünndarm in Form von Passagestörungen unterschiedlicher Genese. Die chirurgischen wie bariatrischen Ergebnisse zeigen, dass das Ausmaß der Frühund Spätkomplikationen im Vergleich zu den Folgen einer fehlenden operativen Behandlung akzeptabel ist.

\section{Einleitung}

Die Kenntnis der möglichen Komplikationen beim laparoskopischen Magenbypass ist Voraussetzung für eine profunde präoperative Vorbereitung und Aufklärung der Patienten, die

\section{Key Words}

Laparoscopic gastric bypass - Bariatric surgery - Complications $\cdot$ Results

\section{Summary}

Postoperative Complications after Laparoscopic Roux-en-Y Gastric Bypass in Bariatric Surgery

The laparoscopic Roux-en-Y gastric bypass surgery involves some characteristic surgical complications besides the general risks associated with bariatric surgery. The overall risk could be effectively decreased due to the minimal invasive technique, though. The mortality is $0-0.5 \%$ and the risk of morbidity varies between 6 and 30\%. However, the specific circumstances of morbidly obese patients make diagnostics difficult. Especially septic complications of any kind represent a high risk of mortality. Therefore, maximal safety und prevention are very important issues to consider. The most common causes of death are pulmonary embolism and insufficiency of anastomosis. Due to the laparoscopic approach complications of wound healing are scarcely observed. The most frequent complications result from pulmonary and cardiac dysfunctions. Surgical complications mainly result from bleedings, problems with the anastomoses and sutures as well as from the small intestine showing any kind of passage malfunction. Overall, the surgical and bariatric results reveal that early postoperative and long-term complications remain within tolerable limits when compared to consequences of a lack of surgery.

sichere Durchführung der Operation, ein rationales postoperatives Management, das rechtzeitige Erkennen drohender oder eingetretener Komplikationen und deren Diagnostik und Therapie sowie selbstverständlich ihrer möglichen Vermeidung.

\section{KARGER \\ (C) 2009 S. Karger GmbH, Freiburg \\ Fax +497614520714 Accessible online at: \\ E-mail Information@Karger.de www.karger.com/ofa \\ www.karger.com}

PD Dr. med. Günther Meyer

Abteilung für Allgemein-, Viszeral- und Gefäßchirurgie

Chirurgische Klinik München-Bogenhausen

Denninger Straße 44, 81679 München, Germany

Tel. +49 89927 94-1600, Fax -1603

dr.meyer@chkmb.de 
Das optimale Komplikationsmanagement beinhaltet eine niedrige Verdachtsschwelle und möglichst frühzeitige Diagnosestellung, die bedingt durch die aufgrund der adipösen Bauchdecken häufig problematische klinische Beurteilbarkeit sehr schwierig sein kann. Da es sich um Hochrisiko-Patienten handelt, ist die frühzeitige Erkennung sich anbahnender oder bereits eingetretener Komplikationen besonders wichtig, denn die nachfolgenden Verläufe sind oft besonders schwer und langwierig und das Mortalitätsrisiko steigt. Die klinischen Symptome, insbesondere bei septischen intraabdominellen Symptomen, unterscheiden sich oft von denjenigen bei nichtadipösen Patienten. Hinzu kommen eine Reihe spezifischer Risiken und Komplikationsmöglichkeiten dieser Operation im frühen wie späten postoperativen Verlauf. Ihre konservative wie operative Therapie muss persönlich und im Umfeld des Krankenhauses beherrscht werden. Der Chirurg sollte daher theoretisch und praktisch entsprechend vorbereitet sein, bevor er diesen Eingriff durchführt.

Wesentlich für die Vermeidung bzw. optimale Diagnostik und Therapie von Komplikationen ist darüber hinaus ein im Umgang mit morbid adipösen Patienten erfahrenes multidisziplinäres Team aus Anästhesisten, Intensivmedizinern, Radiologen, Internisten, Psychologen, ernährungsmedizinischen Fachkräften, Physiotherapeuten sowie entsprechend geschultem Fach- und Pflegepersonal im Operationssaal, auf der Intensiv- und auf der Normalstation. Das Krankenhaus muss über eine spezielle Einrichtung und apparative wie instrumentelle Ausrüstung verfügen. Die konsequente Einhaltung etablierter routinemäßiger Abläufe im postoperativen Verlauf und eine standardisierte Nachsorge sind weitere wesentliche Bestandteile eines auf eine weitestmögliche Vorwegnahme drohender Komplikationen ausgerichteten bariatrischen Programms.

Mit dem Wechsel von der konventionellen zur laparoskopischen Operationsdurchführung konnte neben anderen Vorteilen das Operationsrisiko bedingt durch den minimal-invasiven Zugang verringert werden (Tab.1). Die Magenbypass-Operation sollte heute daher laparoskopisch durchgeführt werden.

Im Folgenden werden die wichtigsten spezifischen postoperativen Komplikationen beim Roux-Y-Magenbypass dargestellt.

\section{Komplikationen}

Weltweit ist der Roux-Y-Magenbypass heute das am häufigsten angewandte bariatrische Operationsverfahren, mit dem nach 1-2 Jahren durchschnittlich ein Übergewichtsverlust von 60-70\% sowie eine Verbesserung oder vollständige Rückbildung der Adipositasbezogenen Komorbiditäten bei $>90 \%$ der Patienten erwartet werden kann [10-12].

Dabei werden in der Literatur Letalitätsraten von $0-1,95 \%$, überwiegend zwischen 0 und $0,5 \%$, angegeben [12-21]. Als unabhängige Risikofaktoren haben sich bei der laparoskopischen Operationsdurchführung die Anastomoseninsuffizienz und die Lungenembolie herausgestellt; bei der konventionellen Operation kamen noch der BMI und die Hypertonie hinzu. In einer Meta-Analyse konnte gezeigt werden, dass die 30-Tage-Letalität nach konven-
Tab. 1. Senkung des Operationsrisikos bei gleicher Effizienz durch laparoskopisches Vorgehen [1-9] ${ }^{\mathrm{a}}$

\section{Vorteile}

Geringerer Blutverlust*

Weniger Schmerzen*

Frühere Mobilisation

Bessere Lungenfunktion, weniger Atelektasen*

Weniger allgemeine Komplikationen

Kürzerer stationärer Aufenthalt*

Schnellere Rehabilitation*

Weniger Wundinfektionen*

Weniger Narbenhernien*

Schnellere Verbesserung der Lebensqualität*

Bessere Kosmetik

Kein Unterschied

Operationsdauer

Anastomoseninsuffizienz*

Kosten*

Übergewichtsverlust*

Nachteile

Längere Lernkurve

Mehr Anastomosenstenosen*

${ }^{a}$ Konversionsrate 2,1-2,5\%.

* Evidenzlevel Ib.

tioneller Operation 0,5\%, nach laparoskopischer Operation dagegen nur $0,2 \%$ betrug und insbesondere die Spätletalität (>30 Tage bis 2 Jahre) mit 1,1 versus $0,1 \%$ bei der konventionellen Operation deutlich höher lag. Weiter zeigte sich ein deutlich höheres Letalitätsrisiko für Männer gegenüber Frauen mit 4,74 bzw. 0,13\% sowie für Patienten mit Super-Adipositas (BMI > 50) mit 1,25\% [22].

Die perioperative Morbidität wird mit einer erheblichen Schwankungsbreite von 6,1-30,3\% angegeben [7, 12, 23]. Dabei treten Minor-Komplikationen in 5-27\% und Major-Komplikationen in 3,3$7 \%$ der Fälle auf $[12,24]$. Die Rate der Frühkomplikationen wird mit 4,2-20,4\% und die der Spätkomplikationen mit 8,1-13,5\% angegeben $[13,14,25,26]$. In einer univariaten Analyse stellten sich ebenfalls nur männliches Geschlecht und Gewicht als signifikante Risikofaktoren für schwere lebensbedrohliche Komplikationen heraus. Patienten $>55$ Jahre zeigten zwar keine höhere Komplikationsrate, jedoch eine dreifach höhere Mortalität als Zeichen einer verminderten Reserve bei Entwicklung einer Komplikation. Interessanterweise ergab sich kein Zusammenhang zwischen einem erhöhten Komplikationsrisiko und den Faktoren BMI, Nikotin, Hypertonie, Diabetes, Osteoarthritis, Schlafapnoe und Notwendigkeit einer Therapie mit CPAP (Continuous Positive Airway Pressure) in der postoperativen Periode [27]. In einer anderen Untersuchung, zwar mit verschiedenartigen bariatrischen Operationen, aber überwiegend Magenbypässen, zeigte sich dagegen ein statistisch erhöhtes Risiko für eine postoperative Sepsis bei Patienten mit Diabetes, Osteoarthritis und Schlafapnoe. Eine zusätzliche präoperative Begleiterkrankung (Diabetes, Hyperlipidämie, Hypertonie, Asthma, Osteoarthritis, Schlafapnoe, gastroösophageale Refluxkrankheit und Depression) war mit einer 24,5\% höheren Wahrscheinlichkeit mit einer Komplikation an der Anastomose und mit einer 23,5\% höheren Wahrscheinlichkeit mit der Entwick- 
Tab. 2. Symptome der Anastomoseninsuffizienz bei Patienten nach Adipositaschirurgie

Tachykardie

Tachypnoe

Fieber/Leukozytose/CRP-Anstieg

Heftiger abdomineller Schmerz

Linksseitiger Schulterschmerz

Singultus

Dyspnoe

Gesteigerte Ängstlichkeit

Absinken des arteriellen $\mathrm{SpO}_{2}$

Veränderung der Sekretion aus Drainage

lung einer Sepsis in den ersten 180 postoperativen Tagen verbunden. Von den untersuchten individuell vorliegenden Begleiterkrankungen stellten sich die Schlafapnoe und die Refluxerkrankung als für die Entwicklung von Komplikationen am meisten prädiktiv heraus [28].

Es handelt sich somit insgesamt um niedrige Letalitäts- und akzeptable Komplikationsraten mit geringer perioperativer Morbidität.

Ein Zusammenhang von Operationshäufigkeit und Komplikationsrate kann vermutet werden. Hierin ist wahrscheinlich auch die Erklärung für die erheblich divergierenden Angaben in der Literatur zu suchen. Zweifellos besteht auch für einen geübten Laparoskopiker mit großer Erfahrung am oberen Gastrointestinaltrakt eine längere Lernkurve für diesen anspruchsvollen Eingriff. In mehreren Studien konnte ein signifikanter Zusammenhang zwischen dem Chirurg- wie Krankenhaus-bezogenen Operationsvolumen und der Komplikations- wie Mortalitätsrate aufgezeigt werden [29, 30]. Dabei zeigten sich in einer Analyse allerdings deutlich geringere Raten schon ab 10 bzw. 34 Operationen pro Jahr pro Chirurg bzw. pro Klinik [31].

\section{Anastomoseninsuffizienz}

Die meist innerhalb der ersten postoperativen Woche auftretende Anastomoseninsuffizienz ist neben der Lungenembolie die häufigste Ursache für Morbidität und Mortalität [32]. Dabei ist die Gastrojejunostomie wesentlich häufiger als die Jejunojejunostomie betroffen. Die Inzidenz wird mit 0-5,1\% angegeben [12, 16, 17, 20, 33-35].

Die Symptomatik ist vielfältig (Tab. 2), wobei besonders auf eine Tachykardie zu achten ist. Postoperativ sollte daher eine routinemäßige Überwachung der Herzfrequenz und der Sauerstoffsättigung erfolgen. Bei den klinisch meist kaum beurteilbaren Bäuchen muss jede sich entwickelnde Tachykardie über 120 Schläge/min den Verdacht auf eine intraabdominelle Komplikation aufkommen lassen und unverzüglich abgeklärt werden. Differentialdiagnostische Ursachen der Tachykardie können eine Lungenembolie, eine Blutung, aber auch starke Schmerzen oder eine Hypovolämie sein. Sind diese Ursachen ausgeschlossen und kann auch mittels Radiologie (Gastrografin-Schluck) [36], durch Methylenblau-Schluck oder endoskopisch [37] keine definitive Klärung erfolgen, empfiehlt sich eine baldige Relaparoskopie zur Revision des Operationsgebietes und gegebenenfalls therapeutischen Intervention. Da Kontrastmitteluntersuchungen bei Insuffizienzen falsch-negative

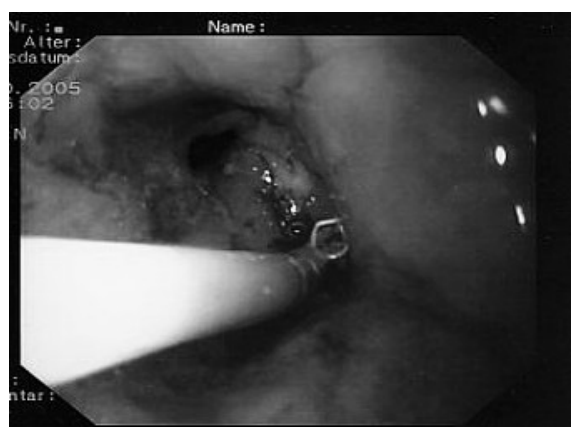

Abb. 1. Endoskopische Fibrinklebung einer Insuffizienz an der Pouch-jejunalen Anastomose.
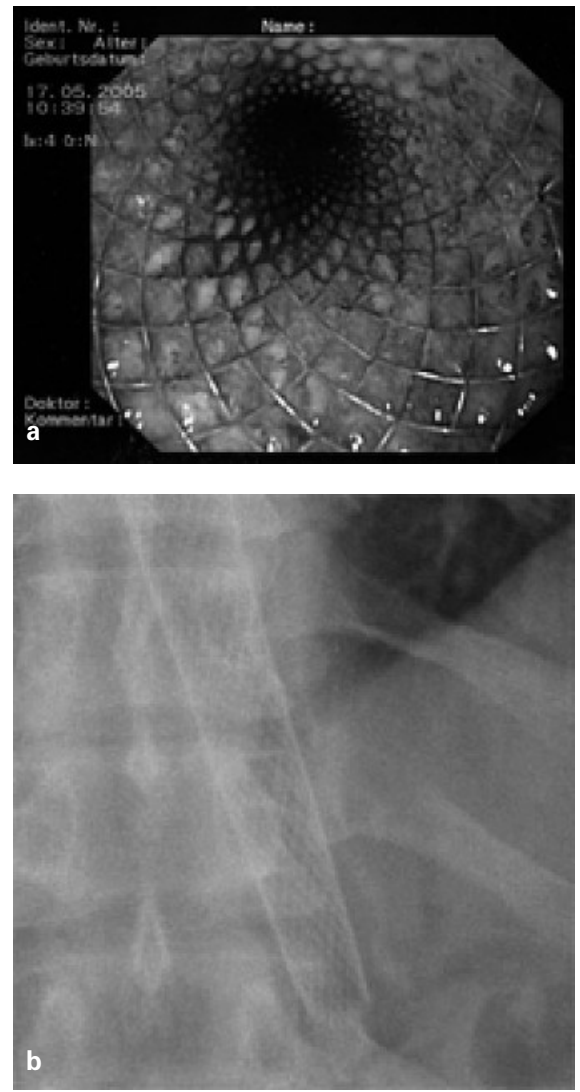

Abb. 2. Endoskopisch über eine insuffiziente Pouch-jejunale Anastomose bis in den abführenden alimentären Schenkel platzierter Covered Stent. a Endoskopische Sicht, b zugehöriges radiologisches Bild.

Ergebnisse zeigen können, sollte bei klinischem Verdacht auf Insuffizienz auch bei fehlendem radiologischem Befund relaparoskopiert werden. Immer muss bedacht werden, dass bei diesen Hochrisiko-Patienten jede septische Komplikation drastische Konsequenzen haben kann und daher möglichst früh behandelt werden sollte. Abhängig vom klinischen Bild und Befund kommen verschiedene therapeutische Möglichkeiten in Betracht: interventionelle Drainage, endoskopische Fibrinklebung, endoskopisches Stenting (covered stent) [37, 38], eine Relaparoskopie oder eine Laparotomie. Endoskopische Interventionen mit Fibrinklebung (Abb.1) oder Stenting (Abb. 2) setzen meist eine suffizient platzierte Drainage voraus. Liegt eine Drainage nicht richtig oder ist sie nicht vorhan- 
den, kann deren Platzierung mittels interventioneller Radiologie, gelegentlich auch sonografisch gesteuert erfolgen. Meist empfiehlt sich jedoch insbesondere bei einer Stentplatzierung eine simultane oder zeitnahe Relaparoskopie mit Spülung des Situs und Drainageneinlage unter Sicht. Insbesondere bei Frühinsuffizienzen bestehen gute Chancen auf einen dauerhaften Verschluss durch eine direkte Übernähung, deren Dichtigkeit möglichst endoskopisch intraoperativ überprüft werden sollte. Im seltenen Falle einer gleichzeitigen Stenose sollte diese endoskopisch dilatiert und der weitere Verlauf bei drainiertem Situs abgewartet werden. Ein nachfolgender Spontanverschluss ist möglich. Prinzipiell sollte bei einer operativen Reintervention aber ein Nahtverschluss der Insuffizienz in Kombination mit Reinigung und Spülung des Situs und Platzierung einer Zieldrainage angestrebt werden. Mit dem Ziel einer möglichst schnellen enteralen Ernährung kann eine Ernährungssonde transnasal tief in den alimentären Schenkel eingeführt werden. Im Falle einer Relaparoskopie oder Laparotomie ist die Platzierung eines geeigneten Tubus bzw. Katheters über eine Gastrostomie eine gute Alternative. Eine begleitende, baldmöglichst testgerechte Antibiotikatherapie ist selbstverständlich.

Das jeweilige Vorgehen wird von individuellen Erfahrungen und dem Zustand des Patienten bestimmt. So konnte in einer Studie mit 40 Nahtinsuffizienzen gezeigt werden, dass bei differenziertem Vorgehen nur bei $10 \%$ der Patienten eine Laparotomie und bei $30 \%$ die laparoskopische Platzierung eines Gastrostomiekatheters erforderlich war und immerhin $60 \%$ der Insuffizienzen erfolgreich nichtoperativ beherrscht werden konnten [39]. Andere vertreten dagegen einen aggressiveren Standpunkt und schlagen eine frühe operative Intervention bei allen Patienten mit Insuffizienzen vor [40].

Zahlreiche intraoperative Maßnahmen sollen dazu beitragen, das Risiko einer Nahtinsuffizienz so gering wie möglich zu halten. Davon haben die nachfolgend aufgeführten eine unumstritten große Bedeutung. Bei der Präparation und Durchtrennung des Magens ist eine Devaskularisation des Pouches unbedingt zu vermeiden. Die Gastrojejunostomie sollte möglichst spannungsfrei angelegt werden. Hierzu hat sich die Durchtrennung des fetten und sperrenden Omentum majus bewährt. Reicht dies nicht aus, kann ein an sich mit Nachteilen behafteter retrokolischer und eventuell sogar retrogastrischer Hochzug der alimentären Schlinge notwendig werden. Grundsätzlich sollten Fixationsnähte zur Entlastung der Anastomose zwischen abführender Jejunalschlinge und Magenpouch gelegt werden. Sie nehmen bei der stets angestrebten Frühmobilisation des Patienten den Zug des schweren Mesenteriums auf die Anastomose. Immer sollte die Anastomose auf Dichtigkeit überprüft werden. Hier stellt die Luftinsufflation unter einem Kochsalzspiegel eine sichere Alternative zur Dichtigkeitsprüfung mit Methylenblau-Lösung dar [41]. Sie wird nach unserer Auffassung am besten endoskopisch durchgeführt [42], wobei die endoskopische Wasserprobe zuverlässiger als der Methylenblau-Test ist [43]. Festgestellte Leckagen müssen seromuskulär sicher übernäht werden. Durch die intraoperative Endoskopie nach Fertigstellung der Gastrojejunostomie kann zusätzlich die ungehinderte Passage in den abführenden Schenkel überprüft und eine etwaige intraluminale Blutung frühzeitig festgestellt werden.
An der enteroenteralen Anastomose sind Kontrollen schwieriger und unzuverlässiger. Vor Verschluss der Brancheninzisionsöffnung sollte aber darauf geachtet werden, dass keine Blutungen aus den Klammernahtreihen der Seit-zu-Seit-Anastomose bestehen. Nach quer fortlaufendem Nahtverschluss der Enterotomie sollte die fertig gestellte Anastomose unter Kompression keine Leckstellen aufweisen [41].

Weitere Empfehlungen werden individuell angewendet. Zahlreiche Arbeitsgruppen übernähen die instrumentelle Nahtreihe prinzipiell mit einer zweiten Nahtreihe [20]. Manche glauben, durch die Verwendung einer Klammernahtverstärkung mit verschiedenen Materialien neben dem Blutungsrisiko auch das Insuffizienzrisiko senken zu können $[44,45]$. Auch durch die zusätzliche Applikation eines Fibrinklebers konnte in einer Studie die Insuffizienzrate vermindert werden [46].

Die Durchführung eines Gastrografinschlucks am 1. oder 2. postoperativen Tag erhöht die Sicherheit zusätzlich. Manche Zentren führen jedoch keine routinemäßige Röntgenuntersuchung mehr durch. In der Abteilung für Allgemein-, Viszeral- und Gefäßchirurgie der Chirurgischen Klinik München-Bogenhausen wird erst mit dem Kostaufbau begonnen, nachdem im Gastrografin-Schluck keine Insuffizienz festgestellt werden konnte. Zudem konnte gezeigt werden, dass 33\% aller Insuffizienzen mit einer Spezifität von $100 \%$ durch die frühe Kontrastmitteluntersuchung entdeckt werden können [47]. Die frühzeitige Diagnose bewirkt eine geringere Morbidität [48] und einen deutlich kürzeren Krankenhausaufenthalt [47]. Der Verzicht auf einen frühen Kontrastmittelschluck führt dagegen erst nach durchschnittlich 7 Tagen zur Diagnose einer Insuffizienz an der Gastrojejunostomie und einer etwa $10 \%$ igen Mortalität [36]. Aber auch bei negativem GastrografinSchluck in den ersten Tagen sollte bedacht werden, dass der Häufigkeitsgipfel des klinischen wie radiologischen Auftretens einer Insuffizienz am 5. postoperativen Tag liegt und daher entsprechende Wachsamkeit geboten ist [12]. Wir führen daher bislang zusätzlich am 5. postoperativen Tag bei noch liegender Drainage einen Methylenblauschluck durch.

\section{Anastomosenstriktur}

Strikturen treten charakteristischerweise nur an der Gastrojejunostomie auf. Ihre Inzidenz wird mit 2-31\% angegeben [35, 49-51]. Unklar ist, warum sie bei Verwendung derselben Anastomosierungstechnik laparoskopisch durchgeführt häufiger auftreten als nach konventioneller Operation.

Symptome entwickeln sich meist innerhalb der ersten 90 postoperativen Tage $[35,52]$ und reichen von der schmerzlosen postprandialen Regurgitation über Übelkeit und Erbrechen bis zur Dysphagie. Die Diagnose ist klinisch meist schnell zu vermuten und wird mittels Gastrografinschluck oder/und Endoskopie bestätigt, wenn ein 9-mm-Endoskop nicht mehr durch die Anastomose vorgeführt werden kann.

Die Therapie der Wahl ist die endoskopische intraluminale Dilatation [51], die wir an dieser Stelle vorzugsweise mit dem Ballon durchführen (Abb.3) und die gelegentlich bis zur dauerhaften Aufweitung, vor allem bei später auftretenden Strikturen, mehre- 

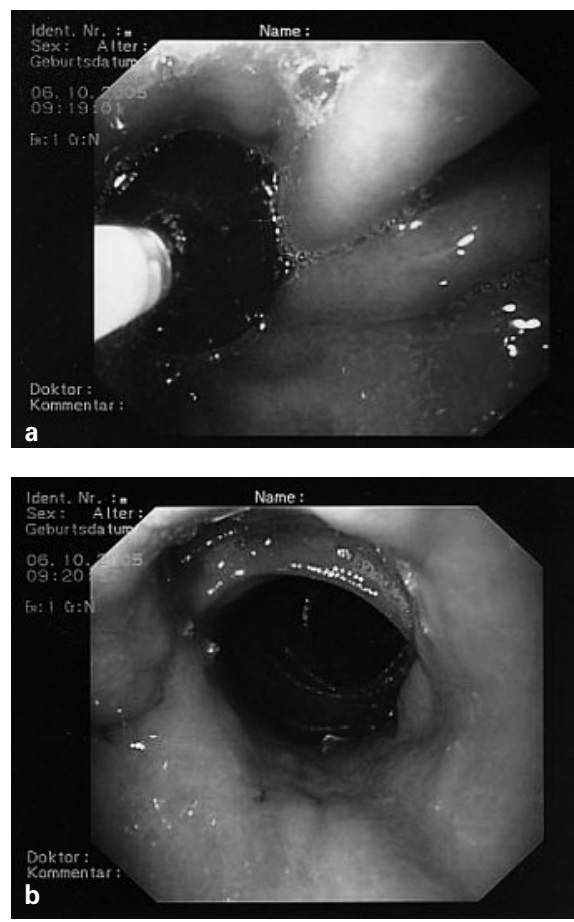

Abb. 3. Endoskopische Ballondilatation einer Striktur an der Gastrojejunostomie. a Ballon in situ, b Situation nach Dilatation.

rer Sitzungen bedarf, letztlich aber so gut wie immer gelingt. Restenosen werden bei 3\% der Patienten beobachtet [53] und können erneut endoskopisch angegangen werden. Eine chirurgische Revision dürfte daher heute kaum noch notwendig werden. Die Dilatationsbehandlung ist ab der 2. postoperativen Woche möglich, erfordert aber zu diesem frühen Zeitpunkt wegen der erhöhten Rupturgefahr ein äußerst behutsames, am besten schrittweises Vorgehen. Später besteht kaum noch ein Rupturrisiko.

Überwiegend besteht die Auffassung, dass das Risiko bei der Verwendung bestimmter Anastomosierungstechniken steigt [35, 51, 52]. Mehrfach konnte gezeigt werden, dass die Strikturrate bei einer Handnaht oder bei Gebrauch eines Linearstaplers deutlich geringer ist als bei Verwendung eines Zirkulärstaplers. Beim Zirkulärstapler ist die Gefahr beim 21-mm-Magazin größer als beim 25-mm-Magazin, ohne dass durch den größeren Anastomosendurchmesser der Gewichtsverlust negativ beeinflusst wird [51].

Weitere präventive Maßnahmen sind die Herstellung einer möglichst spannungsarmen und gut durchbluteten Anastomose. Inwieweit Verstärkungsnähte eine Strikturbildung beeinflussen, ist unklar.

\section{Anastomosenulkus}

Die Inzidenz von Ulzera im Bereich der Gastrojejunostomie variiert zwischen 1 und 16\% [54-57]. Üblicherweise liegen die Ulzera auf der jejunalen Seite der Anastomose und treten in den ersten postoperativen Monaten auf (Abb.4). Sie können sich aber auch erst Jahre nach der Operation entwickeln [58].

Ihre Ursache ist multifaktoriell, aber meist mit der Verwendung nichtabsorbierbaren Nahtmaterials [57] oder einem Pouch-Volumen von mehr als $50 \mathrm{ml}$ verbunden [59]. Auch eine vertikale

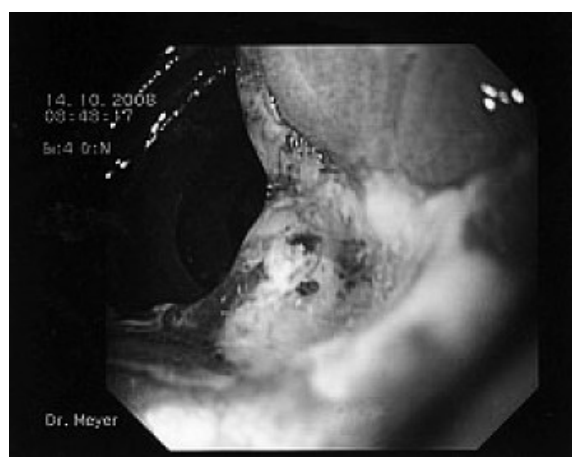

Abb. 4. Anastomosenulkus 8 Monate nach Roux-Y-Magenbypass.

Pouch-Orientierung scheint das Risiko einer Ulkusentwicklung zu erhöhen. Empfohlen wird daher die Herstellung eines schrägen Pouches mit einem Volumen von 15-25, höchstens $40 \mathrm{ml}$ [60]. Von großer Wichtigkeit ist die vollständige Separation von Pouch und Restmagen, um die Ausbildung gastrogastraler Fisteln zu vermeiden. Sie können sich aber auch nach kompletter Abtrennung des Pouches vom distalen Magen entwickeln und treten gehäuft bei Narbenbildungen auf, beispielsweise nach vorausgegangenen Magenbandmigrationen im Gefolge von Redo-Operationen [41]. Klinisch werden diese Fisteln durch das plötzliche Sistieren der Gewichtsabnahme oder eine erneute Gewichtszunahme ersichtlich. Sie gehen oft mit der Ausbildung eines Marginalulkus einher. Deshalb sollte bei der Diagnose eines Anastomosenulkus immer nach einer solchen Fistel gesucht werden. Weitere Risikofaktoren sind die Einnahme nichtsteroidaler Antirheumatika, eine Infektion mit Helicobacter pylori sowie Alkohol- und Nikotinabusus. Im Rahmen der präoperativen Abklärung sollte daher immer ein Helicobacter-Screening durchgeführt werden und bei dessen Nachweis präoperativ eine Eradikationstherapie mit Überprüfung des Ergebnisses erfolgen. Hierdurch kann das Risiko eines Anastomosenulkus signifikant gesenkt werden [61].

Symptome sind Übelkeit, Erbrechen, epigastrischer Schmerz und obere gastrointestinale Blutung.

Die Diagnose wird endoskopisch gestellt. Eine gastrogastrale Fistel kann mit einem Gastrografin-Schluck ausgeschlossen werden. Es sollte eine Untersuchung auf Helicobacter pylori erfolgen.

Die Therapie erfolgt in aller Regel konservativ mit Protonenpumpenhemmern, eventuell in Kombination mit Sucralfat. Bei Patienten mit rezidivierenden Ulzera ist eine kontinuierliche Medikation erforderlich. Im Falle eines Helicobacter-Nachweises wird eradiziert. Noxen wie nichtsteroidale Antirheumatika oder Nikotin sollten vermieden werden. Ulkusblutungen werden primär endoskopisch angegangen. Operative Revisionen stellen die Ausnahme dar und kommen bei chronischen, großen Ulzera, endoskopisch nicht beherrschbaren oder rezidivierenden Blutungen sowie rezidivierenden Ulzera in Kombination mit einem zu großen Pouch-Volumen in Betracht. Hier kann das Problem auch im Rahmen einer Relaparoskopie durch eine Verkleinerung des Pouches mit Exzision des Ulkus gelöst werden. Wird als Ursache für das Ulkus eine zu große Spannung der alimentären Jejunalschlinge mit resultierender Mukosa-Ischämie vermutet, kann dies durch Mobilisation 
der Roux-Schlinge unter vorsichtiger Durchtrennung einer Mesenterialarkade angegangen werden. Fremdmaterial im Ulkus, insbesondere Fäden, können endoskopisch entfernt werden.

\section{Blutung}

Postoperative transfusionspflichtige Nachblutungen sind mit einer Inzidenz von $0,8-4,3 \%$ selten $[17,34]$ und treten bevorzugt an den Klammernahtreihen der Anastomosen und des Restmagens sowie an Milz- oder Mesenterialläsionen auf. Eine operative Blutstillung ist in circa 40\% der Fälle notwendig [39]. Bei intraluminalen Blutungen sollte auch früh-postoperativ der Versuch einer mit der gebotenen Vorsicht durchgeführten endoskopischen Blutungslokalisation sowie nachfolgenden Blutstillung mit Clip-Applikation oder Unterspritzung mit Fibrin-Kleber unternommen werden [37].

Blutungen aus den Staplerreihen sind nicht prinzipiell vermeidbar und kommen häufig vor, weshalb diese immer sorgfältig auf aktive Blutungen überprüft werden müssen. Sie dürfen niemals mit Strom gestillt werden, da dies zu Spätnekrosen mit Leckagen führen kann. Zur Blutstillung eignen sich Clips oder besser eine Umstechungsnaht.

Für die Vermeidung der lästigen und Zeit konsumierenden Klammernahtblutungen ist die Verwendung von Klammernahtmagazinen mit geeigneten Klammergrößen wesentlich: Am Magen sollten blaue Klammernahtmagazine mit 3,5 mm großen Klammern und am Dünndarm weiße Klammernahtmagazine mit 2,5-mm-Klammern eingesetzt werden. Dabei hat es sich bewährt, nach dem Verschluss des Staplers ohne zu großen Widerstand einen Moment zu warten, bis das Instrument schrittweise ausgelöst wird. Seit geraumer Zeit stehen Materialien aus einer absorbierbaren Polymermembran oder Rinderperikard für eine Verstärkung der Klammernaht zur Verfügung. Sie senken das Blutungsrisiko [62] und erhöhen den Berstdruck an der Klammernaht [63]. Obwohl es sich um sehr teure Produkte handelt, dürfte ihr selektiver Einsatz komplikations- und damit kostensenkend sein.

\section{Akute Restmagendilatation}

Die akute Restmagendilatation ist mit einer Inzidenz von $0,6 \%$ [64] zwar selten, aber ein unmittelbar lebensbedrohliches Ereignis. Es erfordert eine sofortige Entlastung des zum Zerbersten prall gefüllten Magens. Ursache ist eine Obstruktion des biliopankreatischen Schenkels, meist bedingt durch ein Problem an der enteroenteralen Anastomose.

Leitsymptom sind stärkste epigastrische Schmerzen in Verbindung mit einer auf der Röntgenübersichtsaufnahme des Abdomens oder in der Computertomographie sofort erkennbaren massiven Dilatation des Magens. Sie führt zu einer rapiden klinischen Verschlechterung des Allgemeinzustands mit Tachykardie und Hypotension sowie Erhöhung von Amylase, Lipase und den Cholestaseparametern im Blut. Letztlich rupturiert die Klammernahtreihe am Restmagen mit sofortiger hämodynamischer Instabilität bis zum sofortigen Herzstillstand. Die massive, raumgreifende Erweiterung des Magens kann die Durchblutung der vor oder hinter ihm ziehenden alimentären Schlinge stören und zu deren Gangrän führen.
Therapeutisch wird bei dramatischer Dilatation sofort eine transkutane, gastrale Punktion mit Drainagen-Einlage zur unmittelbaren Entlastung durchgeführt. Im Anschluss muss die zugrunde liegende Passagestörung operativ beseitigt werden. Ist der Restmagen bereits rupturiert, wird nach Reinigung der Bauchhöhle meist eine Teilresektion zur Entfernung des zerstörten Abschnittes notwendig, um den Magen erneut sicher verschließen zu können.

\section{Innere Hernien}

Während spontan oder postoperativ entwickelte innere Hernien in der allgemeinen Viszeralchirurgie von untergeordneter Bedeutung sind, haben sie mit zunehmend häufiger Anwendung des Roux-YMagenbypasses in der bariatrischen Chirurgie in den letzten Jahren viel Beachtung gefunden. Die Inzidenz zeigt mit 0,5-9\% [6569] sehr unterschiedliche Häufigkeiten. Insgesamt sind innere Hernien die häufigste Ursache einer intestinalen Obstruktion nach Magenbypass.

Durch die Roux-Y-Rekonstruktion beim Magenbypass entstehen insbesondere beim retrokolischen Hochzug Bedingungen, die das Auftreten einer inneren Hernie begünstigen. Deren Entstehung wird zusätzlich durch den Verlust an intraabdominalem Fettgewebe im Rahmen des schnellen postoperativen Gewichtsverlusts und die geringere Tendenz zur Ausbildung von Adhäsionen bei laparoskopischer Operationsdurchführung begünstigt.

Die inneren Hernien nach Magenbypass entstehen typischerweise an 3 Lokalisationen: Am häufigsten entwickeln sie sich nach retrokolischem Hochzug in die Inzision des Mesocolon transversum. Zwischen Mesocolon transversum und dem Mesenterium des hochgezogenen alimentären Schenkels kann sich die so genannte «Petersen-Hernie» ausbilden. Unter die Jejunojejunostomie kann sich eine Dünndarmschlinge zwischen die zugehörigen Mesenterien schieben und sich drehen. Die zugehörigen Häufigkeiten werden mit $67,7,5$ und $21 \%$ angegeben, wobei sich in $4,5 \%$ der Fälle innere Hernien an mehreren Stellen entwickelten [68].

Die Diagnose ist wegen des diffusen klinischen Beschwerdebilds oft schwer zu stellen. Meist klagen die Patienten über intermittierende postprandiale, krampfartige Beschwerden, vorzugsweise periumbilikal im Mittelbauch. Im Vollbild besteht die Symptomatik des Ileus mit Übelkeit und Erbrechen bis hin zum akuten Abdomen bei Entwicklung von Ischämien und Dünndarmnekrosen. Darüber hinaus sind auch die radiologischen Verfahren, wie Gastrografin-Schluck und Computertomographie, unsicher und die Untersucher mit diesem Krankheitsbild wenig bis gar nicht vertraut. Zusätzlich erschwert ist die Diagnostik, wenn der proximale Dünndarm involviert ist und eine zu erwartende Stauung der Darmschlinge klinisch wie auch in der Kontrastmitteluntersuchung fehlt. Die radiologischen Befunde sind deshalb in 20-40\% der Fälle falsch-negativ $[68,70]$. Bei entsprechendem Verdacht bleibt daher meist nur die frühzeitige Durchführung einer diagnostischen Relaparoskopie. Oft kann der Darminfarkt nur durch eine rasche Entscheidung verhindert werden.

Die Therapie besteht in der Reposition, gegebenenfalls einer Resektion und dem Bruchlückenverschluss, d. h. Verschluss des Mesenterialdefekts. Der Eingriff kann häufig übersichtlich und sicher 
im Rahmen einer Relaparoskopie durchgeführt werden. Bei klinisch manifestem mechanischem Ileus nach Magenbypass-Operation empfiehlt sich aber eine konventionelle Reoperation.

Verschiedene Maßnahmen können der Entwicklung dieser Komplikation vorbeugen, die zum Verlust größerer Dünndarmabschnitte bis hin zum Kurzdarmsyndrom führen kann. Bei retrokolischem Hochzug ist die exakte Fixation der Dünndarmschlinge in einem anatomisch optimal gewählten Mesokolonschlitz die beste Prophylaxe [41]. Noch sicherer ist der antekolische Hochzug mit rechtsorientierter Schlinge, wodurch zusätzlich das Risiko der Entwicklung einer Petersen-Hernie reduziert wird [65, 69]. Auch die Anlage eines Short-Limb-Magenbypasses verringert das Risiko der Entwicklung einer inneren Hernie [65]. Die sicherste Prävention stellt aber der antekolische Hochzug mit prinzipiellem Verschluss der Mesoschlitze zwischen Mesocolon transversum und dem Mesenterium der hochgezogenen Schlinge sowie hinter der Enteroanastomose in fortlaufender Nahttechnik mit nichtresorbierbarem
Nahtmaterial dar. Hierdurch kann die Gefahr um 50\% reduziert werden [16]. Andererseits führen zahlreiche Operateure keinen routinemäßigen Verschluss der Mesenterialschlitze am Ende der Operation durch und meinen, kein gehäuftes Auftreten innerer Hernien beobachten zu müssen.

\section{Schlussfolgerung}

Insgesamt finden sich beim laparoskopischen Magenbypass niedrige Letalitäts- und akzeptable Komplikationsraten mit geringer perioperativer Morbidität, die gemeinsam mit sehr guten Langzeitgewichtsverlustraten und überzeugenden Rückbildungsraten der Begleiterkrankungen sowie einer niedrigen Rate an Langzeitkomplikationen dieses Operationsverfahren bei den meisten bariatrischen Patienten derzeit als Methode der Wahl erscheinen lassen.

\section{Literatur}

1 Nguyen NT, Goldman C, Rosenquist J, Arango A, Cole CJ, Lee SJ, Wolfe BM: Laparoscopic versus open gastric bypass: a randomized study of outcomes, quality of life, and costs. Ann Surg 2001;234:279-291.

2 Courcoulas A, Perry Y, Buenaventura P, Luketich J: Comparing the outcomes after laparoscopic versus open gastric bypass: a matched paired analysis. Obes Surg 2003;13:341-346.

3 Westling A, Gustavsson S: Laparoscopic vs open Roux-en-Y gastric bypass: a prospective, randomized trial. Obes Surg 2001;11:284-292.

$\checkmark 4$ Lujan JA, Frutos MD, Hernandez Q, Liron R, Cuenca JR, Valero G, Parrilla P: Laparoscopic versus open gastric bypass in the treatment of morbid obesity. A randomized prospective study. Ann Surg 2004;239:433-437.

5 Marema RT, Perez M, Buffington CK: Comparison of the benefits and complications between laparoscopic and open Roux-en-Y gastric bypass surgeries. Surg Endosc 2005;19:525-530.

6 Smith SC, Edwards CB, Goodman GN, Halversen RC, Simper SC: Open vs laparoscopic Rouxen-Y gastric bypass: comparison of operative morbidity and mortality. Obes Surg 2004;14:7376.

7 Parikh MS, Shen R, Weiner M, Siegel N, Ren CJ: Laparoscopic bariatric surgery in super-obese patients $(\mathrm{BMI}>50)$ is safe and effective: a review of 332 patients. Obes Surg 2005; 15:858-863.

$\checkmark 8$ Lee WJ, Yu PJ, Wang W, Chen TC, Wei PL, Huang MT: Laparoscopic Roux-en-Y versus mini-gastric bypass for the treatment of morbid obesity: a prospective randomized controlled clinical trial. Ann Surg 2005;242:20-28

$\checkmark 9$ Nguyen NT, Lee SL, Goldman C, Fleming N, Arango A, McFall R, Wolfe BM: Comparison of pulmonary function and postoperative pain after laparoscopic versus open gastric bypass: a randomized trial. J Am Coll Surg 2001;192:469-477.

10 Buchwald H, Avidor Y, Braunwald E, Jensen MD, Pories W, Fahrbach K, Schoelles K: Bariatric surgery. A systematic review and meta-analysis. JAMA 2004;292:1724-1738.
11 Wittgrove AC, Clark GW: Laparoscopic gastric bypass: a five-year prospective study of 500 patients followed from 3 to 60 months. Obes Surg 1999;9:123-143.

-12 Schauer PR, Ikramuddin S, Gourash W, Ramanathan R, Luketich J: Outcomes after laparoscopic Roux-en-Y gastric bypass for morbid obesity. Ann Surg 2000;232:515-529.

13 Rosenthal RJ, Szomstein S, Kennedy CI, Soto FC, Zundel N: Laparoscopic surgery for morbid obesity: 1,001 consecutive bariatric operations performed at The Bariatric Institute, Cleveland Clinic Florida. Obes Surg 2006;16:119-124.

14 Parikh MS, Laker S, Weiner M, Hajiseyedjavadi O, Ren CJ: Objective comparison of complications resulting from laparoscopic bariatric procedures. J Am Coll Surg 2006;202:252-261.

15 Shikora SA, Kim JJ, Tarnoff ME, Raskin E, Shore R: Laparoscopic Roux-en-Y gastric bypass. Results and learning curve of a high-volume academic program. Arch Surg 2005;140:362-367.

16 Higa KD, Boone KB, Ho T: Complications of the laparoscopic Roux-en-Y gastric bypass: 1,040 patients - what have we learned? Obes Surg 2000; 10:509-513.

17 Wittgrove AC, Clark GW: Laparoscopic gastric bypass, Roux-en-Y -500 patients: technique and results, with 3-60 month follow-up. Obes Surg 2000;10:233-239.

18 Flum DR, Dellinger EP: Impact of gastric bypass operation on survival: a population-based analysis. J Am Coll Surg 2004;199:543-551.

19 Sekhar N, Torquati A, Lutfi R, Richards WO: Endoscopic evaluation of the gastrojejunostomy in laparoscopic gastric bypass. A series of 340 patients without postoperative leak. Surg Endosc 2006;20:199-201.

20 DeMaria EJ, Sugerman HJ, Kellum JM, Meador JG, Wolfe LG: Results of 281 consecutive total laparoscopic Roux-en-Y gastric bypasses to treat morbid obesity. Ann Surg 2002;235:640-647.

21 Fernandez AZ, DeMaria EJ, Tichansky DS, Kellum JM, Wolfe LG, Meador J, Sugerman HJ: Multivariate analysis of risk factors for death fol- lowing gastric bypass for treatment of morbid obesity. Ann Surg 2004;239:698-703.

22 Buchwald H, Estok R, Fahrbach K, Banel D, Sledge I: Trends in mortality in bariatric surgery: a systematic review and meta-analysis. Surgery 2007;142:621-635.

23 Nguyen NT, Morton JM, Wolfe BM, Schirmer B, Ali M, Traverso LW: The SAGES bariatric surgery outcome initiative. Surg Endosc 2005;19: 1429-1438.

24 Farkas DT, Vemulapalli P, Haider A, Lopes JM, Gibbs KE, Teixeira JA: Laparoscopic Roux-en-Y gastric bypass is safe and effective in patients with a BMI > 60. Obes Surg 2005;15:486-493.

25 Biertho L, Steffen R, Ricklin T, Horber F, Pomp A, Inabnet WB, Herron D, Gagner M: Laparoscopic gastric bypass versus laparoscopic adjustable gastric banding: a comparative study of 1,200 cases. J Am Coll Surg 2003;197:536-547.

26 Weber M, Müller MK, Bucher T, Wildi S, Dindo D, Horber F, Hauser R, Clavien PA: Laparoscopic gastric bypass is superior to laparoscopic gastric banding for treatment of morbid obesity. Ann Surg 2004;240:975-983.

27 Livingston EH, Huerta S, Arthur D, Lee S, De Shields S, Heber D: Male gender is a predictor of morbidity and age a predictor of mortality for patients undergoing gastric bypass surgery. Ann Surg 2002;236:576-582.

28 Cawley J, Sweeney MJ, Kurian M, Beane S: Predicting complications after bariatric surgery using obesity-related co-morbidities. Obes Surg 2007; 17:1451-1456.

29 Weller WE, Rosati C, Hannan EL: Relationship between surgeon and hospital volume and readmission after bariatric operation. J Am Coll Surg;204:383-391.

30 Nguyen NT, Paya M, Stevens CM, Mavandadi S, Zainabadi K, Wilson SE: The relationship between hospital volume and outcome in bariatric surgery at academic medical centers. Ann Surg 2004;240:586-593.

31 Courcoulas A, Schuchert M, Gatti G, Luketich J: The relationship of surgeon and hospital volume 
to outcome after gastric bypass surgery in Pennsylvania: a 3-year summary. Surgery 2003;134: 613-621.

32 DeMaria EJ: Bariatric surgery for morbid obesity. N Engl J Med 2007;356:2176-2183.

33 Schauer PR, Ikramuddin S: Laparoscopic surgery for morbid obesity. Surg Clin North Am 2001;81: 1145-1179.

-34 Marano BJ: Endoscopy after Roux-en-Y gastric bypass: a community hospital experience. Obes Surg 2005;15:342-345

- 35 Gonzalez R, Lin E, Venkatesh KR, Bowers SP, Smith D: Gastrojejunostomy during laparoscopic gastric bypass. Analysis of 3 techniques. Arch Surg 2003;138:181-184.

-36 Marshall JS, Srivastava A, Gupta SK, Rossi TR, DeBord JR: Roux-en-Y gastric bypass leak complications. Arch Surg 2003;138:520-524.

- 37 Meyer G, Merkle R, Schinkel S, Spelsberg F, Weidenhagen R, Schildberg FW: Postoperative Endoskopie zur Diagnose und Therapie von Komplikationen. Chirurg 2002;73:9-21.

38 Eubanks S, Edwards CA, Fearing NM, Ramaswamy A, de la Torre RA, Thaler KJ, Miedema BW, Scott JS: Use of endoscopic stents to treat anastomotic complications after bariatric surgery. J Am Coll Surg 2008;206:935-939.

39 Thodiyil PA, Rogula T, Mattar SG, Schauer PR Management of complications after laparoscopic gastric bypass; in Inabnet WB, DeMaria EJ, Ikramuddin S (eds): Laparoscopic Bariatric Surgery. Philadelphia, Lippincott Williams \& Wilkins, 2005, pp 225-237.

-40 Arteaga JR, Huerta S, Livingston EH: Management of gastrojejunal anastomotic leaks after Roux-en-Y gastric bypass. Am Surg 2002;68: 1061-1065.

41 Weiner RA, Blanco-Engert R, Winterberg U: Laparoskopischer Roux-en-Y-Magen-Bypass Technik und Komplikationen. Chir Gastroenterol 2003;19:46-53

42 Shin RB: Intraoperative endoscopic test resulting in no postoperative leaks from the gastric pouch and gastrojejunal anastomosis in 366 laparoscopic Roux-en-Y gastric bypasses. Obes Surg 2004:14:1067-1069.

43 Ramanathan R, Ikramuddin S, Gourash W, Schauer PR: The value of intraoperative endoscopy during laparoscopic Roux-en-Y gastric bypass. Surg Endosc 2000;14(suppl):S212.

-44 Shikora SA, Kim JJ, Tarnoff ME: Reinforcing gastric staple-lines with bovine pericardial strip may decrease the likelihood of gastric leak after laparoscopic Roux-en-Y gastric bypass. Obes Surg 2003;13:37-44.

45 Shikora SA: The use of staple-line reinforcement during laparoscopic gastric bypass. Obes Surg 2004;14:1313-1320.

46 Liu CD, Glantz GJ, Livingston EH: Fibrin glue as a sealant for high-risk anastomosis in surgery for morbid obesity. Obes Surg 2003;13:45-48.

47 Sims TL, Mullican MA, Hamilton EC, Provost DA, Jones DB: Routine upper gastrointestinal Gastrografin swallow after laparoscopic Rouxen-Y gastric bypass. Obes Surg 2003;13:66-72.

48 Ovnat A, Peiser J, Solomon H, Charuzi I: Early detection and treatment of a leaking gastrojejunostomy following gastric bypass. Isr J Med Sci 1986;22:556-558.

49 Bell RL, Reinhardt KE, Flowers JL: Surgeonperformed endoscopic dilatation of symptomatic gastrojejunal anastomotic strictures following laparoscopic Roux-en-Y gastric bypass. Obes Surg 2003;13:728-733.

50 Goitein D, Papasavas PK, Gagne D, Ahmad S, Caushaj PF: Gastrojejunal strictures following laparoscopic Roux-en-Y gastric bypass for morbid obesity. Surg Endosc 2005;19:628-632.

51 Nguyen NT, Stevens CM, Wolfe BM: Incidence and outcome of anastomotic stricture after laparoscopic gastric bypass. J Gastrointest Surg 2003; 7:997-1003

52 Bradshaw WA, Smith CD: Gastrojejunal stricture following laparoscopic gastric bypass is more likely with circular stapler than hand-sewn anastomosis. Gastroenterology 2001;120(suppl 1): A-491.

53 Ahmad J, Martin J, Ikramuddin S, Schauer P, Slivka A: Endoscopic balloon dilation of gastroenteric anastomotic stricture after laparoscopic gastric bypass. Endoscopy 2003;35:725-728.

54 Sapala JA, Wood MH, Sapala MA, Flake TM: Marginal ulcer after gastric bypass: a prospective 3-year study of 173 patients. Obes Surg 1998;8: 505-516.

55 MacLean LD, Rhode BM, Nohr C, Katz S, McLean AP: Stomal ulcer after gastric bypass. J Am Coll Surg 1997;185:1-7.

56 Pories WJ, MacDonald KG, Flickinger EG, a surgical disease? Ann Surg 1992;215:633-643.

57 Capella JF, Capella RF: Gastro-gastric fistulas and marginal ulcers in gastric bypass procedures for weight reduction. Obes Surg 1999;9:22-28.
Sanyal AJ, Sugerman HJ, Kellum JM, Engle KM, Wolfe L: Stomal complications of gastric bypass: incidence and outcome of therapy. Am J Gastroenterol 1992;87:1165-1169.

59 Jordan JH, Hocking MP, Rout WR, Woodward ER: Marginal ulcer following gastric bypass for morbid obesity. Am Surg 1991;57:286-288.

60 Rasmussen JJ, Fuller W, Ali MR: Marginal ulceration after laparoscopic gastric bypass: an analysis of predisposing factors in 260 patients. Surg Endosc 2007;21:1090-1094.

61 Schirmer B, Erenoglu C, Miller A: Flexible endoscopy in the management of patients undergoing Roux-en-Y gastric bypass. Obes Surg 2002;12: 634-638.

62 Consten ECJ, Gagner M, Pomp A, Inabnet WB: Decreased bleeding after laparoscopic sleeve gastrectomy with or without duodenal switch for morbid obesity using a stapled buttressed absorbable polymer membrane. Obes Surg 2004;14: 1360-1366.

63 Baker RS, Foote J, Kemmeter P, Brady R, Vroegop T, Serveld M: The science of stapling and leaks. Obes Surg 2004;14:1290-1298.

64 Jones KB: Biliopancreatic limb obstruction in gastric bypass at or proximal to the jejunostomy: a potentially deadly, catastrophic event. Obes Surg 1996;6:485-493.

65 Quebbemann BB, Dallal RM: The orientation of the antecolic roux limb markedly affects the incidence of internal hernias after laparoscopic gastric bypass. Obes Surg 2005;15:766-770.

66 Onopchenko A: Radiological diagnosis of internal hernia after Roux-en-Y gastric bypass. Obes Surg 2005;15:606-611.

67 Hwang RF, Swartz DE, Felix EL: Causes of small bowel obstruction after laparoscopic gastric bypass. Surg Endosc 2004;18:1631-1635.

68 Higa KD, Ho T, Boone KB: Internal hernias after laparoscopic Roux-en-Y gastric bypass: incidence, treatment and prevention. Obes Surg 2003;13:350-354.

69 Iannelli A, Facchiano E, Gugenheim J: Internal hernia after laparoscopic Roux-en-Y gastric bypass for morbid obesity. Obes Surg 2006;16:12651271.

Garza E, Kuhn J, Arnold D, Nicholson W, Reddy S, McCarty T: Internal hernias after laparoscopic Roux-en-Y gastric bypass. Am J Surg 2004;188:796-800. 